\title{
LPS-binding protein circulates in association with apoB-containing lipoproteins and enhances endotoxin-LDL/VLDL interaction
}

\author{
Anita C.E. Vreugdenhil, A.M. Patricia Snoek, Cornelis van 't Veer, \\ Jan-Willem M. Greve, and Wim A. Buurman
}

Department of General Surgery, M aastricht University, M aastricht, The N etherlands

Address correspondence to: A.C.E. Vreugdenhil, Department of General Surgery, M aastricht University, PO Box 616, 6200 M D M aastricht, The N etherlands. Phone: 31433881496; Fax: 31433884154; E-mail:W.Buurman@ah.unimaas.nl.

Received for publication July 20, 2000, and accepted in revised form December 5, 2000.

\begin{abstract}
LPS-binding protein (LBP) and serum lipoproteins cooperate in reducing thetoxic properties of LPS. In the present study, we demonstratethat LBP circulates in association with LDL and VLDL in heal thy persons. ApoB was found to account at least in part for theinteraction of LBP with LDL and VLDL. Although LBP interacted with purified apoA-I in vitro, no association of LBP with apoA-I or HDL was found in serum. Consistent with the observed association of LBP with LDL and VLDL, these lipoproteins al so were demonstrated to be the predominant LPS-binding lipoproteins. M ost interestingly, the association of LBP with LDL and VLDL strongly enhanced thecapacity of these lipoproteins to bind LPS. Because this function of LBP is of utmost importance during infection, the association of LBP and LPS with lipoproteins was also studied in serum from septic patients. In septic serum containing high LBP levels and a markedly altered lipoprotein spectrum, most of the LBP is associated with LDL and VLDL, although some LBP appeared to circulatefreefrom lipoproteins. Also in this serum, LPS was found to bind predominantly to LDL and VLDL. The observed binding of LBP and LPS to LDL and VLDL, as well as the LBP-dependent incorporation of LPS into these lipoproteins, emphasizes a crucial role for circulating LBP-LDL/VLDL complexes in the scavenging of LPS.
\end{abstract}

J. Clin. Invest. 107:225-234 (2001).

\section{Introduction}

LPS is a constituent of the outer membrane of gramnegative bacteria and evokes an inflammatory response by activation of monocytes and endothelial cells. LPSinduced cellular responses are the net result of the interaction of LPS with various plasma components such as soluble CD 14, LPS-binding protein (LBP) and membrane receptors such as membrane-bound CD 14 and Toll-like receptors. This initiation of cellular responses is essential for the host defense against bacterial infections. However, if large amounts of endotoxin are present in the circulation, an excessive cellular response can be deleterious for the host, and, therefore, endotoxin-inactivating processes are of extremeimportance.

LPS is detoxified in the circulation by incorporation into lipoproteins (reviewed in ref. 1). Physiological levels of lipoproteins protect against endotoxicity in vitro and in vivo $(2,3)$. Early studies have demonstrated an interaction of LPS with HDL (4); al beit later, also VLDL and LDL were found to bind and inactivate LPS (5-7). Consistent with this, LDL, VLDL, chylomicrons, and HDL all have been observed to reduce the lethal effect of endotoxin in mice (8-10).

Evidence for a physiological rolefor LBP in inflammation is supported by studies that demonstrate enhanced mortality and uncontrolled multiplication and spread of bacteria in LBP knockout micecompared with wild-type mice after intraperitoneal administration of bacteria (11). The results of these studies indicate that LBP is required to induce a rapid inflammatory response, which is essential for the resistance to bacteria. However, LBP has the paradoxical dual function of sensitizing the immune system to endotoxin and, on the other hand, enhancing detoxification of endotoxin. LBP catalyzes the transfer of LPS into lipoproteins, thereby enhancing LPS detoxification (12). Likewise, LBP catalyzes the lipoprotein neutralization of lipoteichoic acid, a component of the cell membrane of gram-positive bacteria (13). Lamping et al. demonstrated in a murine model that high levels of LBP in the circulation, as seen during an acute-phase response, inhibit LPS effects and prevent mortality induced by endotoxemia (14). The latter observation strongly supports a physiological rolefor LBP-dependent detoxification of LPS in the host defense.

Endotoxemia induces an acute-phase response characterized by multiple physiological adaptations. This response appears to play a role in host defense mechanisms, although its physiological relevance needs further elucidation. One aspect of the acute-phase response is a dramatic rise in circulating levels of LBP 
(15). Concomitantly, large changes in serum lipid and lipoprotein concentrations occur. Circulating levels of total cholesterol, LDL chol esterol, and HDL cholesterol decrease, whereas serum triglyceride and VLDL levels increase (16). In addition, alterations in apolipoprotein levels are observed $(16,17)$. ApoA-I concentrations drop, and HDL becomes depleted in apoA-I $(16,18)$. In contrast, apoB levels are not affected by either viral or bacterial infection (16).

Othersfound evidencefor an association of LBP with apoA-I-containing lipoproteins in plasma from healthy persons (12). We consider that the physical association of LBP with these lipoproteins may be important for the cooperation of LBP and lipoproteins in the detoxification of endotoxin. However, thestrong reduction of apoA-I and HDL levels that coincides with the dramatic raise in LBP levels during endotoxemia seems in contrast with this cooperative function. Because it is firmly established that LDL and VLDL are critical in the survival of infection with gram-negative bacteria (19) and that circulating levels of these lipoproteins are relatively high during inflammation compared with HDL levels, the present study was undertaken to investigate whether LBP associates with LDL and VLDL. To this end, the distribution of LBP among lipoproteins was studied in serum of healthy and septic persons. Subsequently, we investigated the effect of the association of LBP with lipoproteins and apolipoproteins on the LPS-binding capacity.

\section{Methods}

Reagents. LPS from Escherichia coli, serotype 055:B5, was purchased from Sigma Chemical Co. (St. Louis, M issouri, USA). Purified apoA-I and apoB were derived from Calbiochem (LaJolla, California, USA) and ICN Radiochemicals Inc. (I rvine, California, USA), respectively. Polyclonal antibodies to human LBP were obtained by immunizing rabbits with purified human LBP. Protein A-purified anti-LBP IgG was biotinylated following standard procedures. Anti-human LBP mAb HM 14 was obtained by immunizing mice with LBP following classical procedures. Horseradish peroxidase-labeled $m A b$ against apoA-I and apoB were gifts from L. Sorell (Center for Genetic Engineering and Biotechnology, Havana, Cuba).

Blood samples. Blood was collected from healthy donors and from septic patients. Informed consent was obtained from healthy donors and relatives of septic patients. To prepare serum, blood was allowed to clot for 2 hours at room temperature. Serum was separated by centrifugation and stored at $-80^{\circ} \mathrm{C}$ until use. Fresh serum was used for isolation of lipoprotein fractions. Lipid electrophoresis and Western blot analysis. Agarose electrophoresis was performed in barbital buffer $(\mathrm{pH}$ 8.6) using the Paragon Lipoprotein Electrophoresis kit $\mathrm{P} / \mathrm{N}$ (Beckman Instruments, Brea, California, USA) according to the manufacturer's instructions. Agarose gel electrophoresis was followed by electrophoretic transfer onto an Immobilon-P membrane (Millipore
Corp., Bedford, M assachusetts, USA) in blotting buffer (25 mM TRIS, $192 \mathrm{mM}$ Glycine, 10\% methanol) using the Phast system (Pharmacia Biotech A/B, Uppsala, Sweden). After transfer, the membranes were blocked with $1 \%$ BSA in PBS for 1 hour at $37^{\circ} \mathrm{C}$ and washed with $0.1 \%$ BSA $0.5 \%$ Tween in PBS. For detection of LBP, membranes were incubated with biotin-labeled polyclonal antibody specific for human LBP, washed, and incubated with peroxidase-labeled streptavidin. After detection of LBP, apoA-I was probed on the same blot after elution of the LBP antibodies by incubation with $0.1 \mathrm{M}$ Glycine $\mathrm{HCl}$ buffer ( $\mathrm{pH} 2.5$ ) for 15 minutes at $37^{\circ} \mathrm{C}$. Localization of apoA-I was followed by detection of apoB in a similar protocol. For immunodetection of apoA-I and apoB, peroxidase-labeled mAb's wereused. Interactions of LPS with serum lipoproteins were studied by preincubation of biotin-labeled LPS with human serum overnight at $37^{\circ} \mathrm{C}$. After preincubation, serum was subjected to electrophoresis and blotted and membranes were incubated with peroxidase-labeled streptavidin. To study LPS binding to isolated (apo)lipoproteins, human serum was first subjected to electrophoresis and blotted and membranes were incubated with biotinylated LPS, followed by incubation with peroxidase-labeled streptavidin (Zymed Laboratories Inc., South San Francisco, California, USA). Peroxidaseactivity was detected by chemiluminescent substrates (SuperSignal Substrate, Pierce Chemical Co., Rockford, Illinois, USA) according to the manufacturer's recommendation.

Lipoprotein fractionation. Lipoproteins were isolated from pooled fresh normal human serum (six healthy donors) by a 22-hour single spin density gradient ultracentrifugation at $200,000 \mathrm{~g}$ and $17^{\circ} \mathrm{C}$ according to Terpstra et al. (20), using a Beckman XL-80 ultra centrifuge with a SW40 rotor and ultraclear centrifuge tubes (Beckman Instruments). A step gradient was constructed from $2 \mathrm{ml}$ serum adjusted to density 1.250 $\mathrm{g} / \mathrm{ml}$ with $\mathrm{KBr}$ and sucrose; two $\mathrm{NaCl} / \mathrm{KBr}$ solutions with density 1.225 and $1.100 \mathrm{~g} / \mathrm{ml}$, respectively; and $0.998 \mathrm{~g} / \mathrm{ml}$ endotoxin-free water. All solutions contained $0.1 \mathrm{mg} / \mathrm{ml}$ EDTA. The lipoprotein fractions VLDL, LDL, HDL2, and HDL3 were collected by aspiration, dialyzed extensively against PBS at $4^{\circ} \mathrm{C}$, and stored at $-80^{\circ} \mathrm{C}$ until use. Cholesterol concentrations in the isolated lipoprotein subfractions were determined using an enzymatic colorimetric test from $F$. Hoffman LaRoche, AG (Basel, Switzerland). The lipoprotein fractions used contained no detectableLBP as assessed by LBP-specific ELISA (15).

Purification of $L B P$ and production of LBP-depleted human serum. LBP was isolated from human plasma by selective-affinity immunosorption as described previously (21). Plasma from heal thy volunteers was kindly provided by thelocal blood bank. In short, anti-hLBP mAb $\mathrm{H}$ M-14 was cross-linked to CN Br-activated Sepharose (Pharmacia Biotech A/S) according to the manufacturer's instructions. Human plasma was applied to the anti-LBP column, and unbound proteins were washed 
out with $0.5 \mathrm{M} \mathrm{M} \mathrm{gCl}$. Bound LBP was eluted with 0.1 M Glycine-HCl buffer (pH 2.5). LBP-depleted serum was produced by passing serum over the anti-LBP column. LBP-depleted serum contained less LBP than $0.05 \%$ of normal serum as assessed by LBP-ELISA.

Preparation of biotin-labeled LBP and LPS. LPS and LBP were biotinylated using hydrazide-biotin (PierceChemical Co.). Briefly, LBP and LPS were oxidized with sodium periodate to generate aldehydes from vicinal diols present on thesaccharideunits. Theproducts werethen made to react with the hydrazidegroups present on the biotin, forming stable hydrazone linkages. Linking biotin to the saccharide units in the LBP glycoprotein using hydrazide-biotin did not alter thebiologic activity of LBP (data not shown).

Assays for evaluating the association of LBP and LPS to (apo) lipoprotein. For detection of complexes of LBP and apoB-containing lipoproteins in serum, an assay was developed. Plates (96-well) were coated with an $\mathrm{mAb}$ against LBP (HM 14). As controls, noncoated plates or plates coated with an aspecific antibody (rat antimurine TN F-R75) were used. Aspecific binding sites wereblocked for 1 hour with 1\%BSA in PBS. Dilution series of human serum were applied and incubated for 1 hour, followed by extensive washing of the plates. Captured apoB was detected using a peroxidase-labeled $m A b$ against $a p o B$ followed by tetramethylbenzidine (TMB) substrate (Kirkegaard \& Perry Laboratories Inc., Gaithersburg, M aryland, USA). The absorbance at 450 $n m$ was determined using an ELISA reader.

For studying the quantitativebinding of LBP and LPS to lipoproteins, an in vitro assay was used. Lipoprotein fractions isolated from pooled human serum werestandardized for cholesterol concentration. N inety-six-well plates werecoated with lipoproteins or apolipoproteins for 2 hours at $37^{\circ} \mathrm{C}$. Aspecific binding sites wereblocked for 1 hour with 1\%BSA in PBS. Plates werewashed with PBS. Concentration ranges of biotin-labeled LBP or LPS diluted in $0.1 \% B S A$ in PBS wereincubated for 1 hour at $37^{\circ} \mathrm{C}$ with theimmobilized lipoproteins or apolipoproteins. Bound LBP or LPS was detected using peroxidaseconjugated streptavidin and TM B.

To determine the effect of LBP association with LDL and VLDL on its capacity to interact with LPS, isolated LDL and VLD L was coated to plates in concentrations of 2 and $14 \mu$ g cholesterol per milligram, respectively. After washing of plates and blocking of aspecific binding sites with $1 \% \mathrm{BSA}$ in PBS, a concentration range of LBP was incubated and allowed to associatewith thelipoproteins overnight at $37^{\circ} \mathrm{C}$. Unbound LBP was washed out, and biotin-labeled LPS was incubated for 1 hour at $37^{\circ} \mathrm{C}$. After washing of plates, bound LPS was detected by addition of streptavidin-peroxidaseand TM B. The LPS-binding capacity of the LBP-lipoprotein complexes was compared with that of lipoproteins free of LBP.

\section{Results}

LBP circulates in association with apoB-containing lipoproteins in healthy persons. Thedistribution of LBP among lipopro- teins in human serum derived from healthy volunteers was evaluated using agarose gel electrophoresis in combination with Western blot analysis. Agarose gel electrophoresis avoids the harsh conditions of several other techniques for separation of lipoproteins, such as high salt concentrations and high gravitational forces encountered in ultracentrifugation or perturbation from polyanion precipitation, which cause loss of associated proteins (22). Accordingly, LBP was not detectable by ELISA (detection limit, $200 \mathrm{pg} / \mathrm{ml}$ ) in the lipoprotein fractions separated by ultracentrifugation. In addition, this techniqueenables thestudy of interactions of serum constituents proportional to serum levels.

Surprisingly, Western blot analysis with an LBP-specific antibody shows that in all subjects investigated, LBP is located only at the $\beta$ (LDL/VLDL) mobility region (Figure 1 ). As a control, LBP-depleted serum derived by selective-affinity immunosorption was used, which lacked reaction with the anti-LBP antibody (Figure 1). I mmunoblotting for apoB demonstrates colocalization of LBP with apoB (Figure 1). LBP was not detected in the $\alpha$ mobility region and did not colocalizewith apo A-I (Figure 1).

FreeLBP, purified from human plasma and devoid of lipoproteins, migrates between the $\beta$ and $\alpha$ regions on agarose gel electrophoresis (Figure 2). We studied whether the $\beta$ mobility of LBP in serum is due to association of LBP with lipoproteins. To this end, lipoproteins werefractionated by ultracentrifugation. Theisolated lipoproteins were preincubated at approximately $50 \%$ of their plasma concentration overnight at $37^{\circ} \mathrm{C}$ with LBP $(30 \mu \mathrm{g} / \mathrm{ml})$ and subjected to electrophoresis, and Western blot analysis of LBP was performed. Preincubation of purified LBP with VLDL or LDL restored the $\beta$ mobility of LBP (Figure 2). This conversion in el ectrophoretic migration did not occur when LBP was preincubated with HDL. These results show that in serum, the association of LBP with LDL and VLDL is responsiblefor its $\beta$-electrophoretic mobility.

Further evidencefor theassociation between LBP and apoB-containing lipoproteins in human serum was found using an assay that specifically and quantitatively detects complexes of LBP and apoB-containing lipoproteins in serum. In this assay, LBP-containing

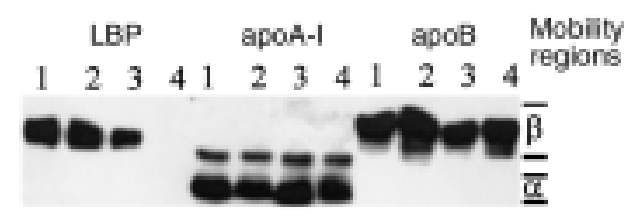

Figure 1

Distribution profile of LBP among lipoproteins in serum of healthy persons. Serum from three healthy persons (lanes 1-3) and LBPdepleted serum (lane 4) were subjected to agarose gel electrophoresis, blotted, and probed with specific antibodies to human LBP, apoA-I, and apoB. LBP colocalizes with apoB in the $\beta$ mobility region and not with apoA-I. 
Figure 2

Effect of LDL and VLDL on the electrophoretic mobility of purified LBP. Purified LBP was preincubated with PBS and isolated LBP-free HDL, VLDL, or LDL (0.1, 0.035 , and $0.5 \mathrm{mg}$ cholesterol/ $\mathrm{ml}$ respectively). Agarose gel electrophoresis of purified LBP and the preincubated fractions was followed by Western blot analysis of LBP using a specific antibody. Purified LBP migrates in between the $\beta$ and $\alpha$ mobility regions. Preincubation of LBP with isolated VLDL or LDL shifts the migration of LBP to the $\beta$ mobility region.

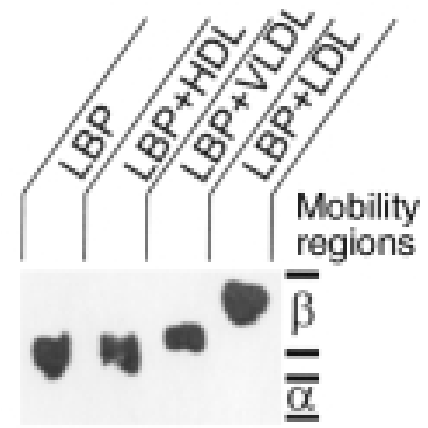

not shown). This suggests that $\beta$-lipoprotein-associated factors account for theLPS binding and that other serum factors are not essentially affecting the distribution.

Thebinding of LPS to lipoproteins was further characterized using an assay similar to that just described for studying LBP-lipoprotein interaction. Binding of LPS to isolated lipoproteins standardized for cholesterol concentration was studied. Also in this assay and fully in line with the results found by Western blot analysis, LDL and VLDL display high LPS-binding capacity under LBP-free conditions, whereas LPS binding to purified $\mathrm{HDL}$ is minor (Figure 6).

LBP bound to lipoproteins enhances the interaction of LPS with lipoproteins. To elucidate whether LBP associated with LDL and VLDL is functionally active in transferring LPS to lipoproteins, we compared LPS binding to lipoproteins with binding of LPS to LBP-lipoprotein complexes. To this end, LDL and VLD L wereimmobi-

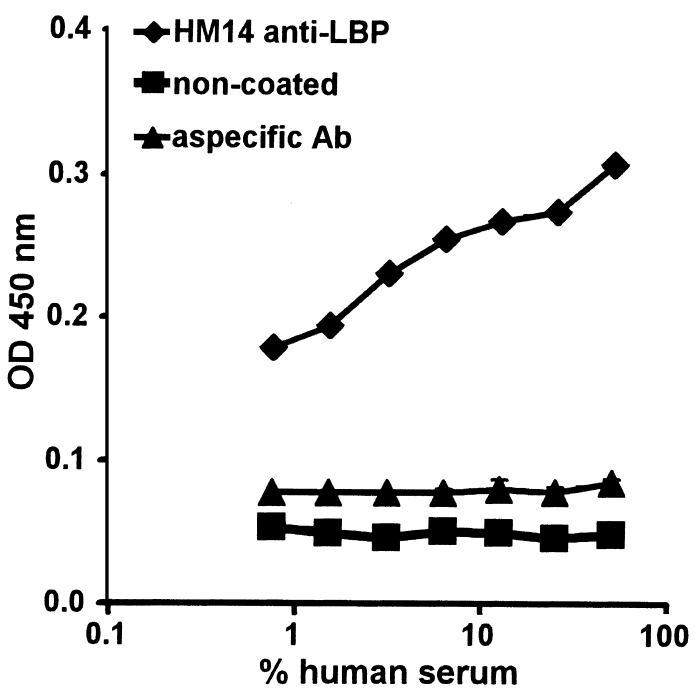

Figure 3

Presence of apoB in LBP-containing lipoproteins. LBP-containing lipoproteins were captured from serum by applying serum to 96 -well plates coated with an mAb against LBP (diamonds) followed by extensive washing of the plates. As a control serum was also applied to noncoated plates (squares) or plates coated with an aspecific antibody (rat anti-murine TNF-R75) (triangles). Presence of apoB in the captured lipoproteins was detected by addition of a peroxidase-labeled $m A b$ against $a p o B$ and is expressed as mean $\pm S D$ of the OD $450 \mathrm{~nm}$ of four wells. 


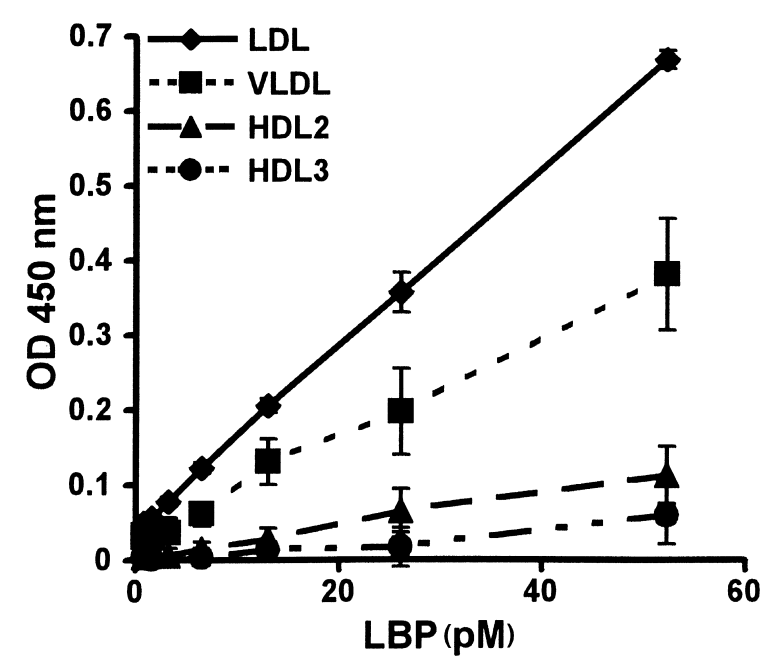

Figure 4

Association of LBP with different lipoprotein classes. Lipoproteins isolated from human serum by ultracentrifugation, free of LBP, and standardized for cholesterol concentration were immobilized to 96well plates and incubated with biotinylated LBP. Bound LBP was detected by peroxidase-conjugated streptavidin and TMB. Binding of LBP to the lipoproteins is expressed as mean \pm SD of the OD 450 $\mathrm{nm}$ of four wells after correction for background. LDL and VLDL display high LBP-binding capacity in contrast to HDL.

lized onto 96-well plates, and a concentration range of LBP was allowed to bind to the lipoproteins for 18 hours at $37^{\circ} \mathrm{C}$. Plates werewashed to removeunbound LBP before biotinylated LPS was added to the preformed LBP-lipoprotein complexes. LBP associated with LDL and VLDL enhanced the binding of LPS to theselipoproteins dose-dependently (Figure 7). These data strongly indicate that LBP bound to LDL and VLD L displays functional properties and enhances the LPS-binding capacity of LDL and VLDL.

Both LBP and LPS associatewith apolipoprotein B. To elucidate whether binding of LBP and LPS to LDL and VLDL depends on interaction with apoB, present in both LDL and VLDL, we studied the binding of LBP and LPS to purified apoB. Contemporaneously, binding of LBP and LPS to purified apoA-I, the predominant apolipoprotein in HDL, was evaluated. Both LBP and LPS bind to apoB dose-dependently (Figure 8, a and b). Binding of LPS to apoA-I was, however, not significant (Figure 8a), which is in accordance with the minor binding of LPS to HDL. Surprisingly, LBP forms a complex with purified apoA-I (Figure 8b).

Because circulating LBP is predominantly associated with lipoproteins containing apoB and not apoA-I (Figure 1), we compared the relative affinities of LBP for apoB and apoA-I. To this end, plates were coated with $a p o B$, and a dilution series of apoA-I and apoB was added to the plates together with biotinylated LBP (Figure 8c). The concentration necessary for $50 \%$ reduction of the signal was $50 \mathrm{nM}$ for apoB and 500 $\mathrm{nM}$ for apoA-I. Theseresults can bedueeither to a difference in affinity for LBP or to the possibility that
apoA-I and apoB have affinity for a different site of LBP. However, when plates were coated with apoA-I, comparable results were found (data not shown): apoA-I concentrations necessary for $50 \%$ reduction of the signal were tenfold higher than apoB concentrations. These data strongly suggest that apoA-I and apoB competefor the same binding site on LBP and that the affinity of LBP for apoB istenfold higher than for apoA-I.

Because lipoprotein-associated LBP enhances the binding of LPS to apoB-containing lipoproteins, we evaluated whether LBP also catalyzes the binding of LPS to the purified apolipoproteins. To this end, plates were coated with apoB and apoA-I, and biotinylated LPS was added to the immobilized apolipoproteins together with $1.7 \mathrm{nM}$ LBP. This LBP concentration was demonstrated to enhance the binding of LPS to LDL and VLDL (Figure 7). LBP enhanced the binding of LPS to apoA-I, whereas it decreased the binding of LPS to apoB (Figure9). These results suggest that theinteraction of LBP with apoA-I enables the binding of LPS to this apolipoprotein, whereas LBP competes with LPS for binding to apoB.

Overall, these data suggest that apoB contains a binding site for both LBP and LPS, and it appears that this binding siteaccounts at least in part for theassociation of LBP and LPS with LDL and VLDL in serum.

LBP and LPS associatewith LDL and VLD L in serum of septic patients. Lipoprotein metabolism, as well as thecomposition of lipoproteins, is altered during the acutephase response. In addition, serum levels of LBP rise dramatically during sepsis (15). Because the data so far indicate that the association of LBP with lipoproteins affects the functional properties of LBP and lipoproteins, which is of utmost interest during endotoxemia, we evaluated in which form theplenitude of LBP produced during sepsis circulates. Serum derived from four septic patients (LBP serum concentration: $121-589 \mu \mathrm{g} / \mathrm{ml}$ compared with $13-16 \mu \mathrm{g} / \mathrm{ml}$ in the heal thy controls) were subjected to agarose gel electrophoresis followed by Western blot analysis. As observed for LBP in serum from healthy per-

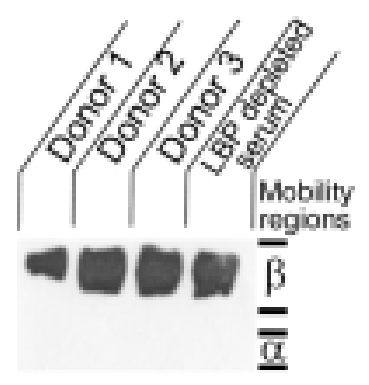

\section{Figure 5}

Distribution profile of LPS among lipoproteins in serum of healthy persons. Serum of three healthy donors and LBP-depleted serum were preincubated with biotinylated LPS. Agarose gel electrophoresis of the sera was followed by Western blotting. Biotinylated LPS was detected using peroxidase-conjugated streptavidin and a chemiluminescent substrate. LPS incubated with serum is recovered in the $\beta$ mobility region. 


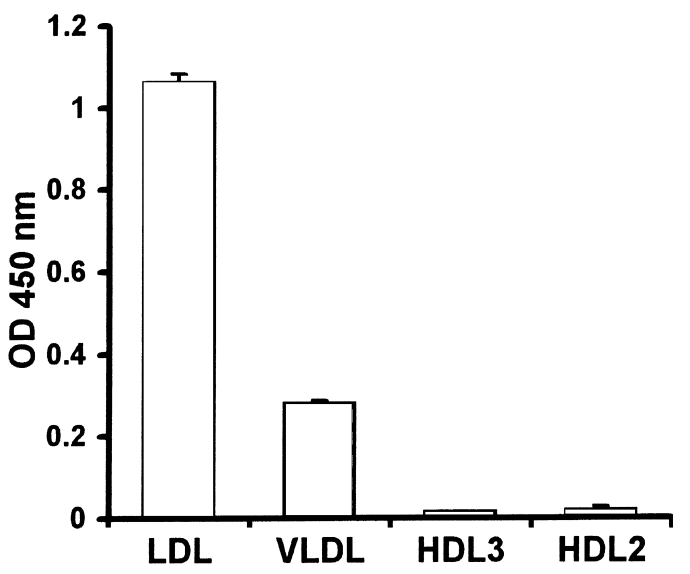

Figure 6

Association of LPS with different lipoprotein classes. Lipoproteins isolated from human serum by ultracentrifugation, free of LBP, and standardized for cholesterol concentration were immobilized to 96well plates followed by incubation with biotinylated LPS. Binding of LPS to the lipoproteins was detected by peroxidase-conjugated streptavidin and TM $B$ and expressed as mean \pm SD of the OD $450 \mathrm{~nm}$ of four wells after correction for background. LDL and to a lesser extent VLDL display high LPS-binding capacity in contrast to HDL.

sons, LBP circulates during septicemia predominantly associated with LDL and VLDL (Figure 10). However, an additional LBP band is observed between the $\beta$ and the $\alpha$ regions, indicativefor the presence of freeLBP. As expected, apoA-I levels dropped markedly in theseptic patients (Figure 10). Also, no association of LBP with HDL was observed in septic patients.

In addition, we investigated whether the alterations in the composition of lipoproteins during infection and the presence of free LBP affected the distribution of LPS among lipoproteins. Biotinylated-LPS preincubated with serum from septic patients was found to migrate with $\beta$-electrophoretic mobility and to comigratewith apoB. Thesefindings are in accordancewith the data obtained from healthy persons and suggest that also during an acute-phase response, LPS binds predominantly to LDL and VLDL (Figure 10).

\section{Discussion}

Thestructure and function of LBP are extensively studied; however, knowledgeconcerning thein vivo forms or associations with other serum components and the effect of these associations on thefunction of LBP is limited. In thepresent study, weobtained evidencethat LBP circulates in association with apoB-containing lipoproteins in healthy persons and in septic patients. This association is functional, as LBP bound to LDL and VLDL was observed to enhance the LPS-binding capacity of theselipoproteins, a process known to result in protection from the deleterious effects of LPS toxicity.

In search of thefactors involved in the association of LBP with apoB-containing lipoproteins in serum, we found that this interaction is at least in part mediated by an interaction of LBP with apoB. Although LBP was not associated with apoA-I or HDL in serum, our experiments demonstrate that LBP does bind to purified apoA-I. Evidencefor a specific interaction of LBP with apoA-I is supported by studies by M assamiri et al., who demonstrated that binding of LBP to reconstituted $\mathrm{HDL}$ is partially blocked by antibodies against apoA-I (23). In the present study, we observed a tenfold higher affinity of LBP for apoB compared with apoA-I (Figure $8 c)$. The amount of apoA-I molecules in serum, however, is about 30 times higher than the amount of apoB under healthy conditions, therefore, it is not possible to subscribe the predominant interaction of LBP with LDL and VLDL to the higher affinity of LBP for apoB. It is possiblethat LBP interacts with domains of apoA-I that are masked by incorporation in native HDL. Furthermore, other constituents than apoB may contribute to the high affinity of LBP for LDL and VLD $L$ and consequently increase the competition for binding of LBP to HDL.

The LBP-transporting lipoproteins LDL and VLDL were also found to be the predominant LPS-binding lipoproteins in normal human serum, an observation which is supported by others $(5,24)$. Studies performed in rodents report the preferential binding of LPS to HDL (4, 25-27). Relatively high HDL levels in rodents seem to account for the predominant binding of LPS to $\mathrm{HDL}$, as high LDL levels in Watanabe heritable hyper-

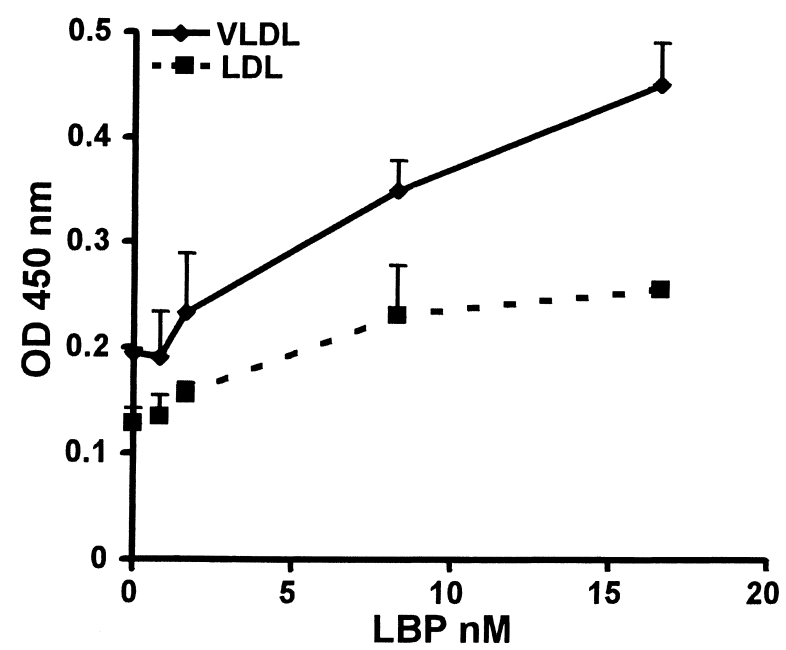

Figure 7

LBP associated with LDL and VLDL enhances the interaction of LPS with lipoproteins. Plates were coated with isolated LDL (2 mg cholesterol/ml) or VLDL (14 mg cholesterol/ml). The immobilized lipoproteins were preincubated with a concentration range of LBP overnight at $37^{\circ} \mathrm{C}$. Unbound LBP was removed by washing the plates, and biotinylated LPS was added to the LBP-lipoprotein complexes. Plates were washed to remove unbound LPS and bound LPS was detected using peroxidase-conjugated streptavidin and TM B. Binding of LPS to the lipoproteins is expressed as mean \pm SD of the OD 450 $\mathrm{nm}$ of four wells after correction for background. LBP associated with LDL and VLDL enhances the binding of LPS dose-dependently. 
Figure 8

Association of LBP and LPS with apoB and apoA-I. (a) Binding of biotin-labeled LPS to apolipoproteins was evaluated. To this end, plates were coated with a concentration range of apoB and apoA-I, and biotin-labeled LPS was added. Bound LPS was detected by peroxidase-conjugated streptavidin and TM B and expressed as mean \pm SD of the OD $450 \mathrm{~nm}$ of four wells after correction for background. (b) Binding of biotin-labeled LBP to immobilized apoB ( $25 \mathrm{nM})$ or apoA-I (100 nM) was detected by peroxidase-conjugated streptavidin and TMB and expressed as mean \pm SD of the OD $450 \mathrm{~nm}$ of four wells after correction for background. (c) The relative affinities of LBP for apoB and apoA-I were evaluated. Biotinylated LBP was added to plates coated with apoB $(25 \mathrm{nM})$. Inhibition of this interaction of LBP with apoB by apoA-I and apoB was studied by adding concentration ranges of apoA-I and apoB to gether with LBP. Bound LBP was detected by peroxidase-conjugated streptavidin and TM B and expressed as mean \pm SD of the OD $450 \mathrm{~nm}$ of three wells after correction for background.

lipidemic rabbits and high VLDL levels in apoE knockout mice result in the predominant binding of LPS to $\operatorname{LDL}(5,28)$ and to VLDL $($. Kuiper personal communication), respectively. In contrast to rodents, the HDL:LDL/VLDL ratio is low in humans, which most likely causes the differences observed for the distribution of LPS among lipoproteins in humans and rodents.

Our data further demonstrate that LBP associated with LDL and VLDL strongly enhances binding of LPS to theselipoproteins in a dose-dependent fashion. However, presence of $L B P$ is not required for theinteraction of LPS with LDL and VLDL. ApoB, present in LDL and VLDL, was demonstrated to have high affinity for LPS and therefore may account for LPS binding under LBPfree conditions. This interaction of LPS with apoB is inhibited by presence of LBP, implicating competition of LBP and LPS for apoB. It can be hypothesized from thesedata that apoB functions as an anchor for LBP on LDL and VLDL. This bound LBP may catalyzethe binding of LPS to other lipoprotein components and there by enhance the total LPS-binding capacity. Given that others found that a lipid-lipid interaction accounts for the association of LPS with lipoproteins (5) and have demonstrated that LBP enhances binding of LPS to phospholipid membranes $(29,30)$, it is likely that LBP linked to apoB enhances intercalation of LPS into the phospholipid membrane of thelipoproteins. Other proteins present in LDL and VLDL may, however, also contribute to the LPS-binding capacity of these lipoproteins. ApoE, present in VLDL, for instance, was demonstrated to bind LPS (31). Most interestingly, apoE-d eficient mice display increased susceptibility to endotoxemia (32), the latter supporting a possible role for apoE in thescavenging of LPS.

During infection, lipoproteins are proven to befundamental for the survival of the host $(1,2,9,10,19)$. Under these conditions, lipoprotein levels and composition are known to be altered profoundly (33). These changes prompted us to evaluate the interaction of lipoproteins with LBP and endotoxins in septic serum. The changes in lipoprotein composition and themore
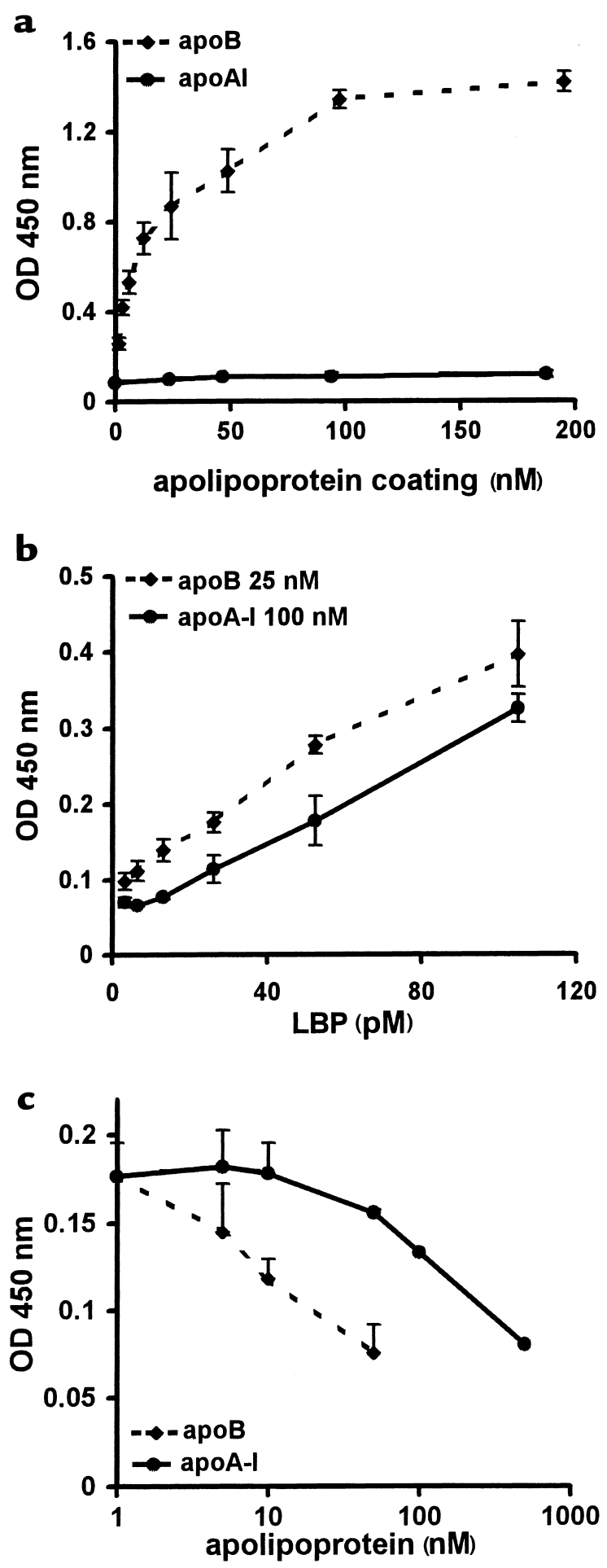

than tenfold enhanced LBP levels did not significantly affect the distribution profile of LBP. In serum of septic patients, LBP is al so colocated predominantly with apoB (Figure 10). Theseobservations indicate that during sepsis, approximately tenfold more LBP is associated with LDL and VLDL. This is of particular interest, as we observed that the LBP-induced upregulation of LPS binding to these lipoproteins is dosedependent. Only a small fraction of thetotal LBP in septic sera migrates 


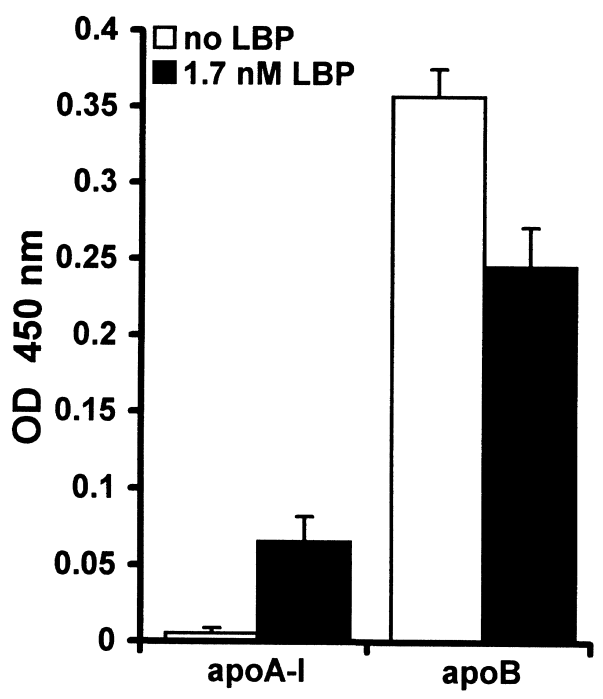

\section{Figure 9}

LBP enhances binding of LPS to apoA-I and reduces binding of LPS to apoB. Plates were coated with apoA-I ( $25 \mathrm{nM})$ or apoB $(100 \mathrm{nM})$. A total of $1.7 \mathrm{nM}$ of LBP was added together with biotinylated LPS, followed by peroxidase-conjugated streptavidin and TM B. Binding of LBP to the apolipoproteins is expressed as mean $\pm S D$ of the $O D$ $450 \mathrm{~nm}$ of four wells after correction for background. The binding of LPS to apoA-I is markedly enhanced by LBP, whereas LBP reduces the binding of LPS to apoB.

as free LBP. Whether there is a difference in biologic activity between lipoprotein-associated and non-lipoprotein-associated LBP in the host response to endotoxin is currently under our investigation. The predominant incorporation of LPS in LDL and VLDL is also observed in septic serum and does not seem to beinfluenced by the al terations in lipoprotein composition and the presence of free LBP. O ur results, which indicatethat LPS and LBP both predominantly bind to LDL and VLDL under septic conditions and that apoB forms a binding site for LBP and LPS, are in line with the findings of others (16) that in contrast to apoA-I, apoB levels stay high during infection. The remaining high concentration of apoB in LDL and VLDL seems functional, as it may contributeto theenhanced binding of LBP and, as a consequence LPS, to theselipoproteins during infection.

It is firmly established that binding of LPS to lipoproteins reduces LPS toxicity (3). In accordance, hypolipidemia results in enhanced LPS-induced lethality in animals (2), and in humans, low levels of cholesterol predict an increased risk of death from infection (34), which emphasizes thesignificance of lipoproteins in protection from bacteria and their toxins. The protectivefunction of lipoproteins is considered to be dueto an increase in the clearance of LPS by formation of LPS-lipoprotein complexes and to prevention of its binding to cells. In addition, it was recently demonstrated that lipoproteins, including LDL, also promotetherelease of cell-associat- ed LPS, which was proved to be dependent on LBP (35). This inhibition of endotoxin binding reduces activation of monocytes and thereby the secretion of cytokines (3, $35,36)$. This protective property of lipoproteins is not only described for gram-negative bacteria, but also the toxic effects of fragments of gram-positive bacteria are inhibited by LDL, a process catalyzed by the presence of LBP (13). Thebeneficial rolefor LDL in thehost defense against bacteria is supported by a study that demonstrates that LDL-receptor-deficient mice with el evated circulating LDL concentrations are protected against lethal endotoxemia and severeinfections with gram-negativemicro-organisms (19).

The uptake of LPS into LDL, which we consider beneficial during acute infection, should be considered as potentially harmful during chronic inflammation. Since we studied LPS binding to LDL and VLDL, and not toxicity, we cannot exclude that LPS binds to LDL and VLDL in a manner by which it retains some toxic activity. In the long term, these complexes may play a pathogenic role in the development of atherosclerosis. Although LDL protects endothelial cells from acute LPS toxicity by formation of LPS-LD L complexes, these complexes migrate across the endothelium and, via unknown pathways, increasethesecretion of monocyte chemotactic activity by endothelial cells (28). As a consequence, transport of LDL-LPS complexes into the artery wall may initiatean inflammatory responseand provoke an atherosclerotic reaction.

In conclusion, we found strong evidence for an association of LBP with apoB-containing lipoproteins in the circulation of healthy persons. Also in septic patients with extremely high circulating LBP concentrations, LBP is predominantly bound to apoB-containing lipoproteins. Most interestingly, LBP associated with LDL and VLDL enhances the LPS-binding capacity of theselipoproteins in a dosedependent manner. Accord-

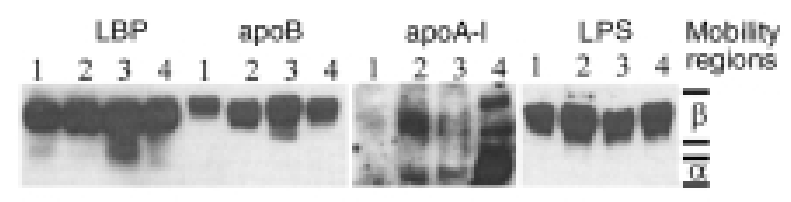

Figure 10

Distribution profile of LBP and LPS in serum of septic patients. Agarose gel electrophorese of serum from four septic patients (lanes 1-4) was followed by Western blot analyses using specific antibodies for LBP, and apoB. Agarose gel electrophorese of serum from three septic patients (lanes 1-3) and a serum pool of healthy persons (lane 4) was followed by Western blot analyses using specific antibodies for apoA-I. LBP predominantly colocalizes with apoB. An additional band compared with normal serum is observed in all subjects between the $\beta$ and $\alpha$ regions, and is most explicit in subject 3 . Preincubation of LPS with the sera followed by Western blot analyses demonstrates the distribution of LPS among lipop roteins during an acute-phase response. LPS colocalizes with LBP and apoB in the $\beta$ region under these conditions. 
ingly, in serum from healthy persons and from septic patients, LDL and VLDL are the predominant LPSbinding lipoproteins. Overall, the data of this study suggest that LBP is a cofactor of circulating LDL and VLDL, which facilitates the uptake of endotoxin by these lipoproteins. This implies an important rolefor LBP/LDL and VLD L complexes in the defense against bacteria and endotoxin.

\section{Acknowledgments}

Wethank M. Poezefor the assistancein obtaining the serum of septic patients. This work was supported by a grant of the Dutch Digestive Diseases Foundation, The N etherlands.

1. Read, T.E., et al. 1993. The protective effect of serum lipoproteins against bacterial lipopolysaccharide. Eur. H eart J. 14:125-129.

2. Feingold, K.R., et al. 1995. Role for circulating lipoproteins in protection from endotoxin toxicity. Infect. Immun. 63:2041-2046.

3. Flegel, W.A., Wölpl, A., M ännel, D.N., and N orthoff, H. 1989. Inhibition of endotoxin-induced activation of human monocytes by human lipoproteins. Infect. I mmun. 57:2237-2245.

4. Ulevitch, R.J., Johnston, A.R., and Weinstein, D.B. 1979. N ew function for high density lipoproteins. J. Clin. Invest. 64:1516-1524.

5. Van Lenten, B.J., Fogelman, A.M., H aberland, M .E., and Edwards, P.A. 1986. The role of lipoproteins and receptor-mediated endocytosis in the transport of bacterial lipopolysaccharide. Proc. Natl. Acad. Sci. USA. 83:2704-2708.

6. Victorov, A.V., et al. 1989. Composition and structure of lipopolysaccharide-human plasma low density lipoprotein complex. Biochim. Biophys. Acta. 984:119-127.

7. N etea, M .G., et al. 1998. Bacterial lipopolysaccharide binds and stimulates cytokine producing cells before neutralization by endogenous lipoproteins can occur. Cytokine. 10:766-772.

8. Read, T.E., et al. 1995. Triglyceride-rich lipoproteins improve survival when given after endotoxin in rats. Surgery. 117:62-67.

9. Harris, H.W., et al. 1993. Chylomicrons alter the fate of endotxin, decreasing tumor necrosis factor release and preventing death. J. Clin. Invest. 91:1028-1034.

10. Harris, H.W., Grunfeld, C., Feingold, K.R., and Rapp, J.H. 1990 Human very low density lipoproteins and chylomicrons can protect against endotoxin-induced death in mice. J. Clin. Invest. 86:696-702.

11. Jack, R.S., et al. 1997. Lipopolysaccharide-binding protein is required to combat a murinegram-negative bacterial infection. Nature. 389:742-745.

12. Wurfel, M .M ., Kunitake, S.T., Lichenstein, H ., Kane, J.P., and Wright, S.D. 1994. Lipopolysaccharide (LPS)-binding protein is carried on lipoproteins and act as a cofactor in the neutralization of LPS. J. Exp. Med. 180:1025-1035.

13. Grunfeld, C., et al. 1999. Lipoproteins inhibit macrophage activation by lipoteichoic acid. J. Lipid Res. 40:245-252.

14. Lamping, N., et al. 1998. LPS-binding protein protects mice from septic shock caused by LPS or gram-negative bacteria. J. Clin. Invest. 101:2065-2071.

15. Froon, A.H .M., Dentener, M .A., Greve, J.W.M., Ramsay, G., and Buurman, W.A. 1995. Lipopolysaccharide toxicity regulating proteins in bacteremia. J. Infect. Dis. 171:1250-1257.

16. Sammalkorpi, K., Valtonen, V., Kerttula, Y., Nikkilä, E., and Taskinen, M.-R. 1988. Changes in serum lipoprotein pattern induced by acute
infections.M etabolism. 37:859-865.

17. Alvarez, C., and Ramos, A. 1986. Lipids, lipoproteins and apoproteins in serum during infection. Clin. Chem. 32:142-145.

18. Cabana, V.G., Siegel, J.N., and Sabesin, S.M . 1989. Effects of the acute phase response on the concentration and density distribution of plasma lipids and apolipoproteins. J. Lipid Res. 30:39-49.

19. N etea, M.G., et al. 1996. Low-density lipoprotein receptor deficient mice are protected against lethal endotoxemia and severe gram-negative infections. J. Clin. Invest. 97:1366-1372.

20. Terpstra, A.H.M., Woodward, C.J.H., and Sanchez-M uniz, F.J. 1981. Improved techniques for the separation of serum lipoproteins by density gradient ultracentrifugation: visualization by prestaining and rapid separation of serum lipoproteins from small volumes of serum. Anal. Biochem. 111:149-157.

21. Vreugdenhil, A.C.E., et al. 1999. Lipopolysaccharide binding protein and serum amyloid $A$ secretion by human intestinal epithelial cells during the acute phase response. J. I mmunol. 163:2792-2798.

22. Kunitake, S.T., et al. 1994. Identification of proteins associated with apolipoprotein A-I-containing lipoproteins purified by selectedaffinity immunosorption. Biochemistry. 33:1988-1993.

23. M assamiri, T., Tobias, P.S., and Curtiss, L.K. 1997. Structural determinants for the interaction of lipopolysaccharide binding protein with purified high density lipoproteins: role of apolipoprotein A-I. J. Lipid. Res. 38:516-525.

24. Eggesbo, J.B., Lyberg, T., Aspelin, T., Hjermann, I., and Kierulf, P. 1996. Different binding of 125 I-LPS to plasma proteins from persons with high or low HDL. Scand. J. Clin. Lab. Invest. 56:533-543.

25. Tobias, P.S., and Ulevitch, R.J. 1983. Control of lipopolysaccharidehigh density lipoprotein binding by acute phase protein(s). J. Immunol. 131:1913-1916.

26. M unford, R.S., Andersen, J.M ., and 1981, J.M .D ietschy. Sites of tissue binding and uptake in vivo of bacterial lipopolysaccharide-high density lipoprotein complexes. J. Clin. Invest. 68:1503-1513.

27. M unford, R.S., H all, C.L., and Dietschy, J.M . 1981. Binding of Salmonella typhimurium lipopolysaccharides to rat high-density lipoproteins. Infect. I mmun. 34:835-843.

28. Navab, M., Hough, G.P., van Lenten, B.J., Berliner, J.A., and Fogelman, A.M. 1988. Low density lipoproteins transfer bacterial lipopolysaccharides across endothelial monolayers in a biologically active form. J. Clin. Invest. 81:601-605.

29. Schromm, A.B., et al. 1996. Lipopolysaccharide-binding protein mediates CD 14-independent intercalation of lipopolysaccharide into phospholipid membranes. FEBS Lett. 339:267-271.

30. Wurfel, M.M., and Wright, S.D. 1997. Lipopolysaccharide-binding protein and soluble CD 14 transfer lipopolysaccharide to phospholipid bilayers. J. Immunol. 158:3925-3934.

31. Rensen, P.C.N., et al. 1997. Human recombinant apolipoprotein E redirects lipopolysaccharide from Kupffer cells to liver parenchymal cells in rats in vivo. J. Clin. Invest. 99:2438-2445.

32. De Bont, N., et al. 1999. Apolipoprotein E knock-out mice are highIy susceptible to endotoxemia and Klebsiella pneumoniae infection. J. Lipid Res. 40:680-685.

33. Auerbach, B.J., and Parks, J.S. 1989. Lipoprotein abnormalities associated with lipopolysaccharide-induced lecithin: cholesterol acyltransferase and lipase deficiency. J. Biol. Chem. 264:10264-10270.

34. Jacobs, D., et al. 1992. Report of the conference on low blood cholesterol: mortality associations. Circulation. 86:1046-1060.

35. Kitchens R.L., Wolfbauer, G., Albers, J.J., and M unford, R.S. 1999. Plasma lipoproteins promote the rel ease of bacterial lipopolysaccharide from the monocyte cell surface. J. Biol. Chem. 274:34116-34122.

36. Cavaillon, J.-M., Fitting, C., H aeffner-Cavaillon, N ., Kirsch, S.J., and Warren, H.S. 1990. Cytokine response by monocytes and macrophages to free and lipoprotein-bound lipopolysaccharide. Infect. Immun. 58:2375-2382. 
